Nach dem Erkalten säuert man mit $25 \mathrm{ccm} 25 \%$ iger Salzsäure an und verfährt nach Jodkaliumzusatz wie oben weiter.

Ein nach diesem abgeänderten Verfahren untersuchtes Präparat ergab 21,9 und $22,2 \% \mathrm{Sb}_{\mathfrak{g}} \mathrm{S}_{9}$, die Kontrollbestimmung $22,2 \% \mathrm{Sb}_{\mathrm{g}} \mathrm{S}_{\mathrm{g}}$.

Danach wäre die günstigste Versuchsanordnung folgende:

Zirka 0,2 g der f eingepulverten Spießglanzprobe werden genau gewogen in einen Erlenmeyerkolben von $100 \mathrm{ccm}$ Inhalt gebracht und mit einem Gemisch von $5 \mathrm{com}$ offizineller Natronlauge und $10 \mathrm{ccm}$ Wasser auf dem Drahtnetz 2 Minuten lang gekocht. Nach dem Verdünnen mit $10 \mathrm{ccm}$ Wasser filtriert man in einen Glasstopfen-Erlenmeyerkolben von ca. $200 \mathrm{ccm}$ Inhalt (Jodzahlkolben) und wäscht zweimal mit je $10 \mathrm{ccm}$ heißem Wasser nach. Zu dem Filtrat gibt man $25 \mathrm{ccm}$ offizinelle Wasserstoffperoxydlösung zu und kocht nochmals ca. 15 Minuten. Hierauf säuert man die Flüssigkeit mit $25 \mathrm{ccm} 25 \%$ iger Salzsäure an, läßt erkalton, fügt 1-2 g Jodkalium hinzu und titriert nach dem Verdünen mit $25 \mathrm{ccm}$ Wasser nach 5 Minuten das ausgeschiedene Jod mit $1 / 10-N$.-Thiosulfat und Stärke. Gegen Schluß der Titration schüttelt man nach jedem Thiosulfatzusatz krüftig durch.

$1 \mathrm{ccm} 1 / 10-\mathrm{N}$.-Thiosulfat $=0,006 \mathrm{~g} \mathrm{Sb}=0,0084 \mathrm{~g} \mathrm{Sb}_{2} \mathrm{~S}_{\mathrm{z}}$.

Mitteilung aus dem Pharmazeutischen Institut der Moskauer Universitat.

Direktor: Prof. W. T i ch o mirow.

\title{
Zur Untersuchung der Entwickelungsgeschichte von Thea chinensis Sims.
}

Von Mag. pharm. E. B is c h mann.

(Eingegangen den 1. VII. 1914.)

Meines Wissens ist eine Untersuchung der Fintwickelungsgeschichte der Teepflanze noch nicht unternommen worlen. Ich habe jedenfalls in der hierhergehörigen Literatur keine darauf bezüglichen Angaben gefunden. Da ich nun in den Besitz geeigneten Materials kam, habe ich die Lösung dieser Aufgabe zu unternehmen versucht. Leider wies mein Material, wie es sich später bei der Arbeit herausstellte, empfindliche Lücken auf. Es ist wohl aber jedem, der auf fremde Mithilfe bei der Materialheschaffung angewiesen gewesen ist, bekannt, wie schwer es fällt, in allen Hin- 
sichten hefriedigend bedient zu wionden. Ich habe nach einem vergeblichen Versuche die Lticken moines Materials auszufüllen, clauselbo einsehen musen, und veröffentliuhe daher moine Arbeit schon jetzt. Auf die sehr reiche Literatur über Tee und Teepflanze kann ich hier nicht eingehen und verweise Interensenten z. B. auf den Artikel „Tee" von Profersor W. M i t la c h er (,Roal-Enzylopädio der ger. Pharmazie").

Die Blute besteht aus funf Kelchblattern, funf bis noun Blumonblätter (ich fand sochs bis acht), einer großen Anzahl Stauhblätter (ich fand bis 235) und einenı oberständigen, drei- manchmal vierfiwherigen, dicht hehaarten Fruchtknnten. Jedes Fach onthalt vior, manchmal funf anatropo Samenunlagen, wolcho 2 woi ubereinander angeordnote Etagen bilden. Jede Etage bertoht aus zwoi, manchmal drei nebeneinander gelagerten Bamenanlagen.. Wonn vier Fächer vorhanden sind, bosteht oine Dtage in solchem Falle aus einer einzelnen Samenanlage. Die lange der Bamenanlagen ist horizontal, wie das auch aus den bokannten Atlassen von Dr. O. C. Berg und C. F. Soh midt (herausgegeben durch Professor A. Mover und Professor K. Schumann), von Köhlor und von Profossor O. W. T bo mó (nach H. Zi ip pel) zu ersehen ist. Die Form der Samenanlagen int aher in den angefuhrten Atlassen nicht riohtig wiedergegeben, wenigetens war die Form, die in Fig. 1 abgehildet ist; tn moinem Material die beständige. Die Ent.wickelung der Samenanlagen ist die ühliche. Zuerst wölben sich die oberen Schichten des an dio nuch engon; spaltonformigen Fücher grenzenden Gewobes vor. Almahlich wahson diese Vorwölbungen in kleine Hörner aus. Auf diesen Hörkern wrecheinen zuerst die inneren, spater die auBeren Integumente in Form' von sekundären Höckern. Dieselben wawhsen stark aus und umgeben den kleinen Nucellus von allen Seiten. Das Cowebe des Nucellng bloiht einige Zeit undifferenziert, dann abor geht die Entwickelung rasch vor sich. Im fortigen Zustando besitet dio Samenanlage den in Fig. 1 angegebenen Bau.

Das dußere Integument einer solchen Samenanlage besteht aus ca. acht Zellreihen, das innere aus sa. sechs. Die Zotlen der Epidermis sowohl des inneren, als auch 'ies außeren Integothentes liegen sonkrectst arP den darunter gologenen: Zollen. Bin CrofuBhündel durchzieht den Funioulus und das außere Integument und endet an der Basis des Nucellus. Dio Chalaza macht sich bereits hemerkbar.

Der sich schnell entwickelnde Embryoseck verdrungt das Gowebe des Nucellus, so dab in den fortig ontwickelten Bamen- 
anlagen, nur weaige, der Chalaza benschbarte Zellraihen alo Resto des iNucollus rorhanden wind. An der Basie sind dio Antipodon sichtbar, an der entgegengesetriten Beito - die Fizolle und dio Synargidon.

Die drai, den firuohtknoten bildeaden Curpellhlëtter var. wwchsen in jungen Éntwickelungsstadien dessellien nur sur Hälfto. Die andero Hälfte eines jeden Fruchtblattes ist froi und endet in einen nach außen, gebogenen \%ipfel. Der Griffel ist.nouh'nicht auegebildet.., Spätor verwächst der Fruchtknoten auch in seiner uberen Hälfte; es bildet sioh ein.langer Criffel, wolcher' mit einer dreiteiligen Narbe endet. In fertigen Zuetande besitzt, dar Stompol

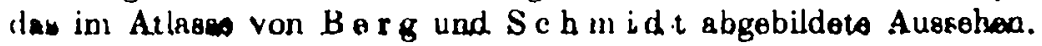
(Ln den Atlageou von $K$ ö h ler und ron $Z$ i i p p l sind die Griffel wioht genïgand anoh mben gebogen 1 f

: Die' Ent wiokelung der Gewebo des Fruchtknotens ist in allgenginen .Zügen ,folgende: Das noch embryonalen Charakter tragende Gewobo hat noch kein Prokambium ausgebilder; rowohl Haare als extah. Krystalle fohlen. In den Blutenstielen sind aber boreits die charakteristiachen Idioblastea vorhanden. Bald aber fangen einzalno Epiderminzellen an sich otwas nach außen vorzustrooken. In diesem Stadium der Entwicholung troten Pror kanhiumatränge auf, obenso Galciumoxalatkrystalle in Gößoror Menge, und awax in den peripheren Geweben des Fruabtknotens: Verhołzte Elomente fehlen noch.

Dio: hintwickelung goht rasch weiter. 'Zahlroicho Yrokambium. stränge hilden einon größeren, äußeren Ring (nach außen von den Semonanlugen) and einen etwas unregelmuBigeren inneren King (nowh. indon. vun don Bamenenlagen). Dio Anzthbl der Caloiunuxalatkrystallo (Drusen) bat sich nooh vermehrt, und zwer liegen dioselbon nach eußen von dem äußeran Ringe der Prokambiumstränge. Auch nach innen zu von dem inneren Ring sind nun roiohlich Cakciumuxalatkryatalle z.u finclou. Danm sind sie auch vereinzelt in den ührigen Partien dos Fruchtknotens un\%utreffen.

. Die Epidormiszellen wacheen bald zu. langen llaaren uus, die anfange dio Ligninreaktionen nicht geben.

. Im.-fertigen .Zustande zeigt das Gewobe des Fuchtknotens dasselbe, Bild, haben siob aber nun Gefaßbündel entwickelt, auoh, geben nun dio, Hurre die Ligninneaktion. V'iele Zellon des Fruobtknotons wend nun mit einem eigentümlichen yolbbraunou Inhalt angefüllt, welcher sehr an ätherisches Oel erinnert. Besonder bäufig fioden sich die borretfenden Zellen in den zentralen Qowehen des Fruchtinotens, in deu die Fücher trennonden Gewoben, in den 
7ellen der Epidermia, in den Zellen, welche die Fächer auskleiden: in den Fpidermiszollen des äußeren Integumentes und in den Zellen der inneren Epidermis des inneren Intogumentes. Auf den Charakter des Inhaltstoffes kommo ich später zurück.

Blliten in den ersten Stadion der Fntwickelung fehlten mir; ehenso junge Früchte, so daß ioh leider wichtige Mơmente der Entwickelungageschichte nicht verfolgen konnte.

Der jungste Samen, den ich in einer Frucht vorfand (in einer und derselben Frucht waren Samen in versohiedenen Entwickelungestadion vorhanden), war $0,6 \mathrm{~cm}$ lang und $0,6 \mathrm{~cm}$ breit. Fr hatto dio gewöbnliche Form der reifen Samen, nar auf dem einen Pole war eine wulstartig hervortrotende Kappe aufgestülpt, welube die später zu beschroibende $z$ weite Schicht mit ihren charakteriatisch entwickelten Zellen aufwies. Pie Farbe de Samens war braun; die Farbe der Kappe - gelbbraun. Die Samenschale besteht in diesom Stadium aus vier Zellsohichten.' Die erste Schicht wird' ton' oiner einreihigen Epidermis gebildet. Die Zellen dersolben énthaiten fast sämtlich den bereits erwăhoten gelbbraunen Thhaltsstoff. Dorselbe sah, wie gesagt, dem ätherischen Oele rhnlich, aber sowubl Alkanna, als auch Sudan färbten äußerst sohwach. Jod furbte gelb, Hämatoxylin - violett (mit bräuntichem Tone), Eisenchlorid - schwärzlich-gran. Eosin färbt ebenfalle. Der er: wähnte Inhaltsatoff enthielt also wohl vorzliglich Gerbstoff, welcher', wie bekannt, oft in Samenschalen vorkommt.

Die 2 weite Schicht hestand aus einigen Roiben stark verdickter und verholzter Zellen, deren Winde mit zahlroichen foinen Puren durchsetzt waren. Auch diese Zellen enthielten oft den bo sohriebenen Inhaltsatoff, ebenso große Calciumoxalatkryibtalle. Diese 2 weite, die Sklerenchymschicht, war aber, wio 'bereits' angedentet, in dem jungen Samen, den ich untersuchte, nooh nicht vollkonmen entwickelt. Sie war nur in der beschriebenen Kappe vorhanden; im librigen bestand diese Schicht noch aus unverhodzten Zellen, zwischen welchen nur vereinzelt die verholzten, chiartikeristischen, vorkamen. Die Verholzung dieser Schicht hatte also erst begonnen. Diese noch unverholzten Zellen der zwoiten Schicht waren noch reicher an dem beschriebenen gelbbraunen Inhalt.

Die dritte Schicht bestand aus zahlreichen Reihen von Parenchymzellen, welche bosonders reich an dem gelbbraunen Inhaltsstoffe waren; dann waren hier GefäBbundel und Calciumoxalatkrystalle vorhanden.

Die vierte Sohicht bestand aus einer, stellenweise auch aus zwei Roihon von Zellen, welche in ihror Form an Palissadenzellen 
erinnerten. Auch diese Zellen führten Gerbstoff. Die nach innen gewandte Wand dieser Zellen gab die Holzstoffreaktion, nicht aber auch die radialen Seitenwände und die nach außen gea ichtete Wand dieser Zellen.

Es ist wohl sehr wahrscheinlich, daß diese vierte Schicht aus der inneren Epidermis des inneren Integumentes hervorgeht. Ich konnte z. B. auch an reifen Samenanlagen beobachten, wie die betreffenden Epidermiszellen eine radiale Lagerung erhielten, wobei sie sich in die Lüngo streckten. Die stufenweise Entwickelung der betreffenden Epidermiszellen zu den Zellen der vierten Schicht habe ich aber leider nicht verfolgen können wegen Mangels an geeignetein Material.

An die vierte Zellschicht der Samenschale schließt eine Schicht teilweise stark zusammengefallener Zellon an, welche aber noch große, Gerbstoff führende Zellen, einzeln oder in Gruppen geordnet, enthielt. Die Zellen dieser Schicht gaben durchweg die Holzstoffreaktion mit. Phloroglucin und Salzsäure.

Da nun, meiner Ansicht nach, alles dafuir spricht, daß die vierte Subicht aus der Epidermis des inneren Integumentes entsteht, so karn die eben beschriebene Schicht nur ein in Stadiun der Obliteration befindliches Endosperm vorstellen. Etwas größere Samen (Länge 0,6 cm, Breite 0,8 cm) zeigten folgendes Bild: Die Epidermiszellen sind, besonders nach KOH.-Bohandlung, gut zu sehen. Die Sklerenchymachicht nimmt jetzt die ganze Peripherie der Samenschale ein. Viele Zellen derselben enthielten Gerbstoff. Die dritte Schicht war unverändert. Weder in der zweiten, nooh in der dritten Schicht waren aber Calciumoxalatkrystalle anzutreffen. Die vierte Schicht war nicht mehr zu eruieren, sogar nach Behandlung mit Kalilauge. Ich erkläre mir diєses scheinbare Verschwinden der vierten Schicht dadureh, da $\beta$ in don vorher beschriebenen, jüngeren Samen die Zellen dieser Schicht meist nur zur Hälfte (und zwar die nach innen gerichtete Hälfte der Zellen) mit dem beschriebenen Inhaltsstoffe gefüllt waren; hei dem weiteren Wachstum der angrenzenden Gewebe war also wohl die leere Hälfte der Zellen zusammengefallen.

Diejenige Zellschicht, welche ich für ein kollabiertes Endosperm ansehe, war nun endgültig zusammengefallen. Die großen, Gerbstoff führenden Zellen waren nicht mehr vorhanden. Phloroglucin und Salzaäure tärben diese Schicht schwach rosa.

Nun gehe ich zur Beschreibung reifer Samen über. Die Form der Samen laängt davon ab, ob eine oder mehr Samenanlagen (ich fand, ungeachtet der entgegengesetzten Angaben, oft zwei, einmal 


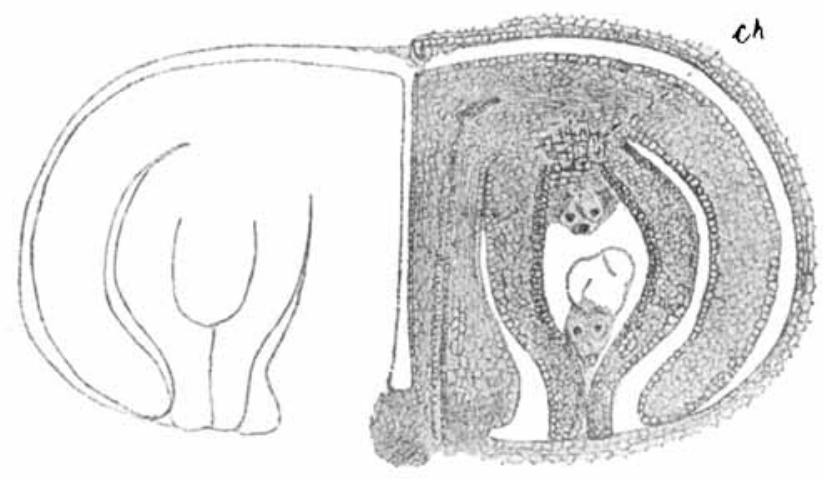

1.
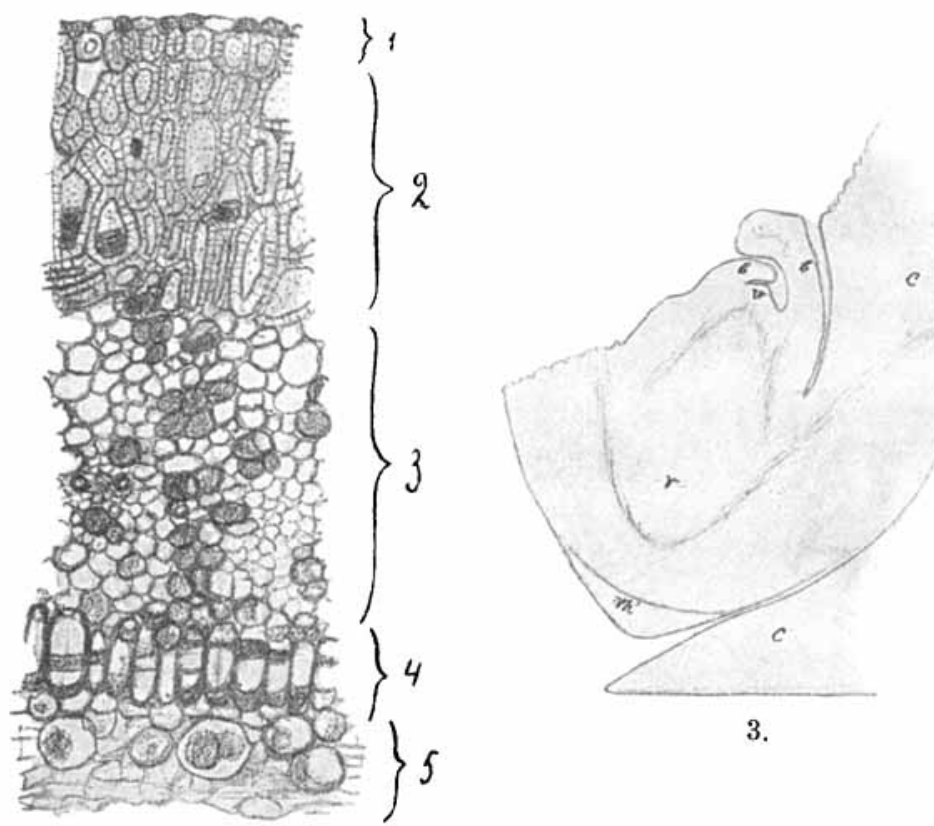

2. 


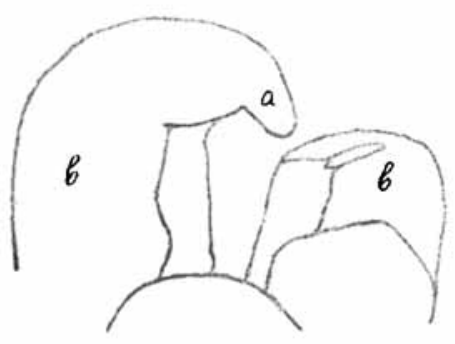

4.

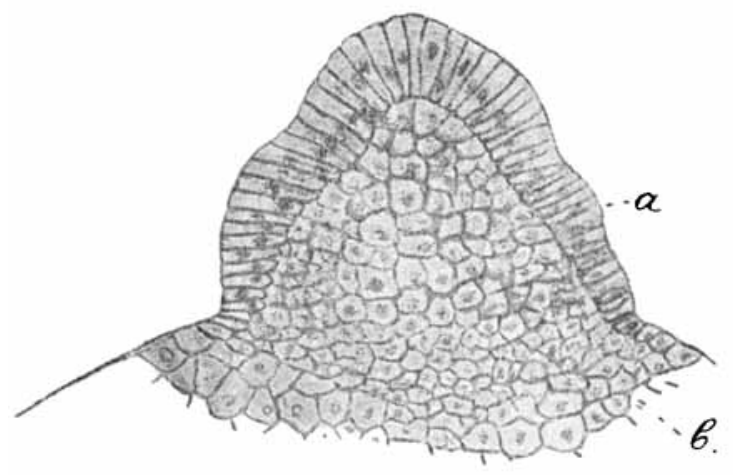

6.

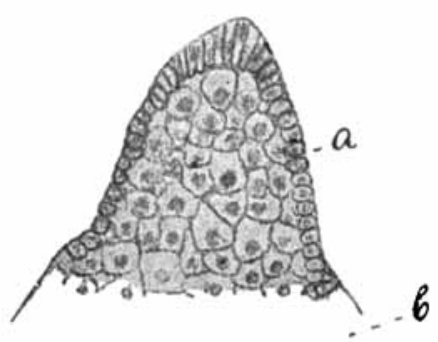

5.

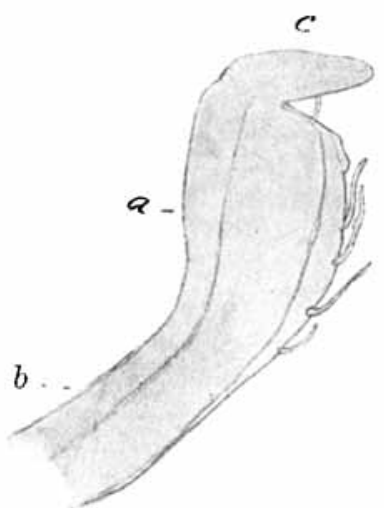

7.

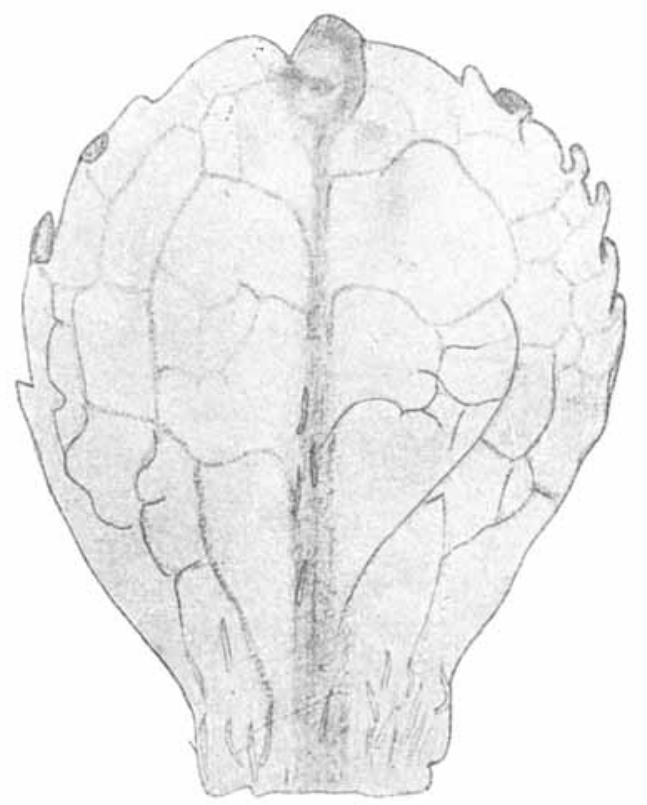

8. 
sogar drei Samen in einem Fache) zur Entwickelung gekommen sind. Ist im Fache nur ein Samen vorhanden, so hat derselbe sich frei und unbeengt entwickeln können; seine Form ist dann kugelförmig mit einer etwas abgeflachten Seite. Haben sich zwei Sumenanlagen entwickelt, so schneiden sich zowöhnlich zwei Seiten des Samens unter seharfem Winkel, die dritte, der zugeschärften Kante gegenüberliegende Seite ist gewölbt. Auf der Sanenschale s!nd die Abdrücke der nicht zur Entwickelung gekommenen Samenanlagen vorhanden. Die Farbe der Samenschale ist hellbraun. Das Hilum hat die Form einer ovalen, flachen Vertiefung, an deren Rande manchmal die Mikropyle in Form eines Punktes zu sehen ist. An dem der Mikropyle gegentiberliegenden Rande des Hilum orhelot sich in Form eines etwas herüberhängenden Höckers der Anfang der Raphe, welche sich dann in Form eines wulstartigen Stranges fortsetzt. Dle Raphe endet an der Chalaza, welche einen mehr oder weniger deutlichen Höcker bildet. Gewöhnlich tritt nur der Anfang der Raphe deutlich hervor; ihre Fortsetzung verliert sich meist auf der Oberfläche des Samens. Auf der Innenseite der Samenschale sind die Gofäßbündelstränge gut zu verfolgen. Der Keim ist von einem dünnen, strukturlosen Häutchen umgeben, welches weder mit der Samenschale noch mit dem Keim verwachsen ist. Dieses Häutchen ist das kollabierte Endosperm.

Der Keim hesteht aus zwei dicken, plankonvexen Kotyledonen, aus einer geraden, dicken, zwischen den Kotyledonen gelegenen Radicula, welche die Form eines nierlrigen Konus besitzt, aus dem undeutlich abgesetzten Hypokotyl und aus der Plumula, welche aus vier Iretibblattunlagen, die den Vegetationskegel umgeben, besteht:

Die mikroskopische Untersuchung der reifen Samen zeigte, daß die Samenschale gebildet wird von einer einreihigen, gut erhaltenen Epidermis, 'von' der' Sklerenchymsuhicht, welche aus ca. 12 Zellreihen besteht (ca. sechs Zellreihen siud radial angeordnet, die ührigen Zelreihen tangential), unk von der vielreihigen dritten Schicht, welche aus lockerem Parenchym, welches von Gefäßbündel durchzogen wird, hesteht. Viele Zellen enthalten Gerbstoff. Die vierte Zellschicht ist nicht zu eruieren.

Die Kotyledonen sind von außen von einer einreihigen Epidormis begrenzt: Das übrige Gewebe besteht aus Parenchymzellen, deren Volumen von der: Peripherie zum Zentrum hin zunimmt. Aus den Kotyledonen tritt je ein diekeres, moeh nicht vollkommen differenziertes Gefäßbündel in die Radikila: Beim Eintritt in die Radikula trennt sich ein sehwicheres GefkBbrindel $a b$, welches in 
die Plumula tritt. Die Zellen dor Kotyledonen sind reich an fettem Oel und an Stärke. Letztere besteht aus kleinen Körnern, welche einen zentralen Bildungskern besitzen. Gewöhnlich sind die Stärkekörner einfach, es kommen aber auch oft ganz zusammengesetzte vor (aus zwei, drei, sogar aus vior Teilkörnern bestehend.

Aleuroukörner habe ich nicht beobachten können, Mill o n s Reaktiv färbte aber die Schnitte bei einigem Stehen rötlich. Nach dem Auswachsen der Samen zeigten die Kotyledonen dasselbe Bild.

Das Gewebe des Embryo des Samens von 0,6 cm Länge und $0,8 \mathrm{~cm}$ Breite hatte den gewöhnlichen embryonalen Bau, und zwar bestand die Radikula aus einem einreihigen Dermatogen und einem mehrreihigen Periblem. Das ringförmig angeordnete Prokambium liegt an der undeutlich ausgerlrückten Grenze zwischen Plerom und Periblem. In dem nach oben gelegenen Teile des Prokambiums hat die Differenzierung bereits angefangen; in dem Prokambiumstrange, der aus dem Kotyledon in dio Radikula tritt, sind boreito enge Spiralgefäße vorhanden.

Bei roifen Samen wird die Peripherie der Radikula von zwei, manchmal drei radjal gelagerten Zellen eingenommen, welche mehr oder weniger deutlich dio Form der bekannten Benzolformel besitzen. Dann kommen ca. 15 Reihen Parenchymzellen, an welche eine Schicht englumiger Zollen, das Prokambium, angrenzt. Das Zentrum wird von dem Markparenchym eingenommen.

Auf Längsschnitten sieht man, daß die Zellen des Radikulagewebes um so mehr an Längo zunehmen, je nähor sie der Spitze zu liegen.

Die Spitze der Radikula wird von einer Wurzelkante bedeckt (siehe Fig. 3). Stärke ist reichlich vorhanden in der oberen Hälfte der Radikula, und zwar in den Geweben, welche das Prokamhium von innen und von außen umgeben. Nach unten zu nimmt der Gehalt an Stärke ab. und zum SchluB versohwindet die Stärke vollkommen aus dem Gewebe. In der Wurzelhaube ist aber Stärke wieder in großer Menge vorhanden. Auch fottes Oel ist reichlich vorhanden, und zwar hauptsächlich in dem peripheren Teile der Radikula. Die Wurzelhaube enthält ebenfalls viel Fett.

Der Vegetationskegel besitzt gewöhnlichen Bau. Die vier Laubblattanlagen sind in verschiedener Größe und verschiedenen Entwickelungestadien vorhanden, und zwar umschließen die älteren Blättchen die jüngeren. Die jüngsten gind noch ganz undifferenziert, noch ist nicht die geringste Andeutung da, die an die fur das Teeblatt charakteristischen Irlisen erinnert. Etwas ältere Blättchen enden aber bereits in eine eigentümliche, zugesuhärfte Spitze aus: 
Dieselbe besteht aus Grundgewebe, das von Epidermiszellen eingerahmt wird, und zwar nehmen hier die Epidermiszellen allmählich an Länge zu, bis sie an dem Spitzenscheitel ihre gröBte Längc erreicht haben; dann nimmet ihre Länge allmählich wieder ab und geht schließlich in die normale Foum tiber (siehe Fig. 5). Das ist der Bildungsanfang der Drlisen des Teeblattes.

Bei noch zilteren Blattchen ist die Spitze nooh deutlicher ausgeprägt und differenzieren sich die sie bedeckenden Epidermiszellen noch deutlicher von den übrigen Epidermiszellen (siehe Fig. 6).

Auoh die älteston der Blättchen der Plumula besitzen nur eine an ihrer Spitze befindliche Drüвe.

Nach dem Auswachsen des Samens entwickelt sich die Radikula bedeutend sohneller, als der oberirdische Teil der Pflanze, und zwar strockt sie sich stark in die Länge. Die Entwickelung ist die gewöhnliche. Leider habe ich eine Norm ftir die Zahl der CefäBteile in der jungen Wurzel nicht feststellen können, da ich in meinem, hierfür nicht sehr reichen Material sieben bis 2 wölf hake zählen können. Das Mark ist reich an Stärke, und zwar sind bier die Stärkekörner ca. viermal so groß, als in der Radikula. In der Rinde ist ebenfalls Stärke vorhauden, aber viel weniger als im Mark.

Die obere Region der jungen Hauptachse ist mit den in charakteristischer Woise gekrümmten Haaren versehen. Mit Phloroglucin und Salzsäure geben dieselben die Ligninreaktion. AeltoreiTeile der Hauptachse sind unbehaart: Aus dem Prokambium, aus dem, wie gewöhnlieh, sich die Gefäßteile und die Siebteile herausdifferenzieren, entwickelt sich nach außen zu ein geschlossener Sklerenchymring, welcher aus ein bis zwei Reihen verholzter Zellen besteht und von dem O. H o y er in seiner Mitteilung in der ,Zeitschrift des Allgem. österr. Apotheker-Vereins" No. 10, 1912, spricht.

Die Zellen des Markes, ebenso - wie die ein-, manchmal zwoireihigen. Markstrahlen verholzen mit der Zeit. Die verholzten Zellon des Markes und der Markstrahlen sind reichb an Stärke. Jüngere Rinde enthält ebenfalls Stärke, allerdings weniger als das Mark, später aber verliert sich diselbe volkommen aus der Rinde. Der kollerichymatische Charakter der peripheren Teile der Rinde ist bereits früh ausgebildet: Ein zwejjärriger Ast voin ca. $0,45 \mathrm{~cm}$ Durchmesser zeigte folgendes 'mikroskopische Bild: Das nicht sehr umfangreiche Mark beștand aus großea, dunnwandigea Zellen; welche die Ligninreaktionen gaben, upd aus kleinen, stark verdickten und ebenfa!ls verholzten Zellen, welche vertikal gelagerte Reihon bildeten. Letztere Zellen enthielten Stärke. Der breite Holzring ist ron Markstrahlen durchzogen, die ebenfalls Stärke 
führten. An den Holzring grenzt die Rinde, deren innerer Ring von typischem Kollenchym gebildet wird. Dieses Kollenchym enthält die für den Tee chaıakteristisehen Idioblasten und Calciumoxalatkrystalle. An das Kollenchym grenzt ein aus ca. fünf Zellreihen bestehender Ring, dessen Zellen verdickt sind, welche die Ligninreaktion geben und sich mit Sudan färben. Weiter nach außen liegt das Periderm, in welchem die Elemente des nun nicht mohr geschlossenen Sklerenchymringes zu finden sind.

Nuch dem Auswachsen strecken sich dis Blättchen und ihr Bau wird deutlicher. Das erste, jüngste, Blättchen ist noch wenig differenziert; hei dem zweiten und besonders dem dritten setzte sich aber die Isamina bereits deutlich ab (siehe Fig. 7). Bei dem vierten Blättchen hebt sich eine gleich unter der Epidermis befindliche Zellreihe von den darunter liegenden Zellen ab. Es ist die sich entwickelnde Palissadenschicht. Dieselbe ist also bei ihrem Entstehen einreihig. Später, bei weiterer Entwickelung, kann die Palissadenschicht aus zwei Reihen von Zellen bestehen, wie das z. B. hei meinem Material der Fall war (mein Material stammte aus Tschakwa, Kaukasus). Professor T i c h o m i r o w hat übrigens in seinem aus China mitgebrachten Materiale nur einreihiges Palissadenparenchym angetroften.

Alle diese Blättchen besaßen je eine Drüse an ihrer Spitze.

Das fünfte Blättchen (von oben) hatto bereits ein gut gewickeltes Gefäßbüudelsystem und denn besa $B$ os bereits acht Drüsen, eine an der Spitze, die übrigen an dem Blattrande (siebe Fig. 8).

Die Blättchen sind hehaart. Im Anfang ist die Behaarung recht unregelmäßig, später konzentriert sich dieselbe hauptsächlich auf den Hauptnerv.

\section{Erklïrungen zu den Abbildungen.}

Fig. 1. Ein Fach des Fruchtknotens mit zwei Samenanlagen; ch $=$ Chaluze.

Fig. 2. Semensehale.

Fig. 3. $v=$ Vegetationskegel; $b=$ Laubblattanlagen; $r=$ Radikula; rh $=$ Wurzelhoube; $\mathbf{c}=$ Kotelydon.

Fig. 4. $\quad a=$ Drüso; $b=$ Leubblattanlagen.

Fig. 5. $a=$ Drüвe der Spitzo einer Laubblattanlage (b).

Fig. 6. a und $b$ wie bei Fig. 5 .

Fig. 7. Des dritte Blättchen eines auswachsenden Semens; $c=$ Drüse; $\mathrm{a}=$ Lamina; $\mathrm{b}=$ Stengel.

Fig. 8. Die Oberseite des fünften Bláttchens. 